\title{
Persistent Tyrosinemia Associated with Low Activity of Tyrosine Aminotransferase
}

\author{
STURE ANDERSSON, ${ }^{(39)}$ ANTAL NEMETH, JORMA OHISALO, AND BIRGITTA STRANDVIK \\ Department of Paediatrics, Karolinska Institutet, Huddinge University Hospital, Sweden [A. N., B. S.] and \\ Department of Medical Chemistry, University of Helsinki, Siltavuorenpenger 10, 00170 Helsinki 17, Finland
}

$[S . A ., J . O$.

\section{Summary}

A son of related Turkish parents had grossly elevated serum tyrosine concentration and excreted tyrosine and $p$-hydroxyphenolic acids into the urine, whereas neither succinylacetone nor succinylacetoacetate could be demonstrated. The tyrosine concentration was normalized by a proper diet. This was not followed strictly at home. During the first 2 years of life, the patient had severe undulating nystagmus that disappeared later. No skin lesions were present and there was only slight corneal clouding of the eyes. At the age of 5 , the patient had attained the maturity of a 4-year-old, showing a balanced profile.

Specific tyrosine aminotransferase (EC 2.6.1.5) was present in the liver; the $K_{m}$ value for tyrosine was normal. However, the total activity was less than $10 \%$ of normal, a situation similar to that observed in fetal human liver. A younger sister of the patient also has tyrosinemia and low hepatic tyrosine aminotransferase activity.

\section{Abbreviation}

pHPP, $p$-hydroxyphenylpyruvate

Tyrosine, the precursor amino acid of thyroid hormones, catecholamines, melanin, and tyramine, is catabolized in the liver via a pathway beginning with transamination in the cytosol by I-tyrosine:2-oxoglutarate aminotransferase (EC 2.6.1.5). This reaction yields pHPP which is then sequentially oxidized by pHPP dioxygenase (EC 1.13.11.27) and homogentisate 1,2-dioxygenase (EC 1.13.11.5) to yield homogentisate and maleylacetoacetate, respectively. The latter compound is sequentially attacked by maleylacetoacetate isomerase (EC 5.2.1.2) and fumarylacetoacetase (EC 3.7.1.2) with final cleavage to fumarate and acetoacetate. Tyrosine aminotransferase is the rate-limiting step of this sequence $(11,13,30)$, and its activity is controlled in a complicated fashion. It is induced by glucocorticoids, glucagon, catecholamines, and tyrosine in rat liver $(2,5,19,25,27,29)$ and in fetal human liver in organ culture $(3,6,13)$.

The metabolism of tyrosine is impaired in liver cirrhosis $(7$, $26)$ and in $30 \%$ of premature newborns $(9,17)$. The significance of these types of tyrosinemia has been under dispute $(17,24)$. Two types of hereditary tyrosinemia have been described in the literature. The more common type, tyrosinemia I, manifests itself initially by failure to thrive, jaundice, hepatosplenomegaly, hematuria, and edema. If the patient lives, severe nodular liver cirrhosis, Fanconi syndrome, and hypoglycemia occur; hepatomas are common $(17,36)$. Tyrosine, pHPP, $p$-hydroxyphenyllactate, succinylacetoacetate, and succinylacetone are excreted into the urine $(10,17)$. This disease is inherited in an autosomal recessive fashion and is due to deficiency of fumarylacetoacetase $(8,22)$.
Tyrosinemia II (Richner-Hanhart syndrome, tyrosinosis of Oregon type) is rare; only a few cases have been described in the literature $(14,16,21,33)$. This syndrome is characterized by mental retardation, nystagmus, and palmar and plantar hyperkeratosis and erosions; clouding, erosions, and dendritic ulcerations of the cornea have been described. Tyrosine and excessive amounts of pHPP are excreted into the urine (14). This disease has been reported to be caused by an absolute deficiency of hepatic tyrosine aminotransferase (14). The excretion of pHPP, the product of the missing enzyme, has been explained by transamination by the mitochondrial isoenzyme of aspartate aminotransferase (EC 2.6.1.1) in extrarenal and extrahepatic tissues that do not have pHPP dioxygenase activity $(14,20)$. This mitochondrial isoenzyme of aspartate aminotransferase that can transaminate tyrosine only at unphysiologically high concentration $(25,28)$ can be separated from tyrosine aminotransferase by virtue of its high pI (31). In three cases, tyrosine aminotransferase activities have been measured in liver biopsy samples and have been reported to be totally absent in the cytosol $(14,18,21)$.

In the present investigation, we describe two siblings with persistent tyrosinemia and partial deficiency of cytosolic tyrosine aminotransferase.

\section{CASE REPORTS}

The first patient was the son of healthy Turkish parents. The father and the maternal grandmother were first cousins. The paternal grandmother (not related to the mother) had a suspected metabolic disease. The patient had two younger sisters, one of which will be described below. The other sister was apparently healthy.

The boy was born at term in normal delivery after an uncomplicated pregnancy and was appropriate for gestational age (3340 $\mathrm{g}, 52 \mathrm{~cm}$ ). A moderate unconjugated jaundice resolved spontaneously at the age of 1 month. Routine neonatal screening revealed elevated serum tyrosine levels while the concentrations of other amino acids were normal. The child was hospitalized at 1 month of age. A mild hepatosplenomegaly was found without any further clinical symptoms. Administration of vitamin $\mathrm{C}$ did not influence serum tyrosine levels. A diet free of tyrosine and low in phenylalanine was introduced at the age of 1 month. However, it was not followed strictly at home (Fig. 1). The levels of serum tyrosine in relation to diet are indicated in the figure. Introduction of this diet always resulted in a normalization of serum tyrosine levels in 3-4 days.

An intensive undulating nystagmus of sudden onset arose at the age of 5 months after 3 months of normal diet and high serum tyrosine levels. The nystagmus lasted for $1 \frac{1 / 2}{2}$ years and faded away slowly. From the second year on, the boy adopted a strange, torticollis-like posture when concentrating on near objects. During his toddler years, the boy had frequent febrile 


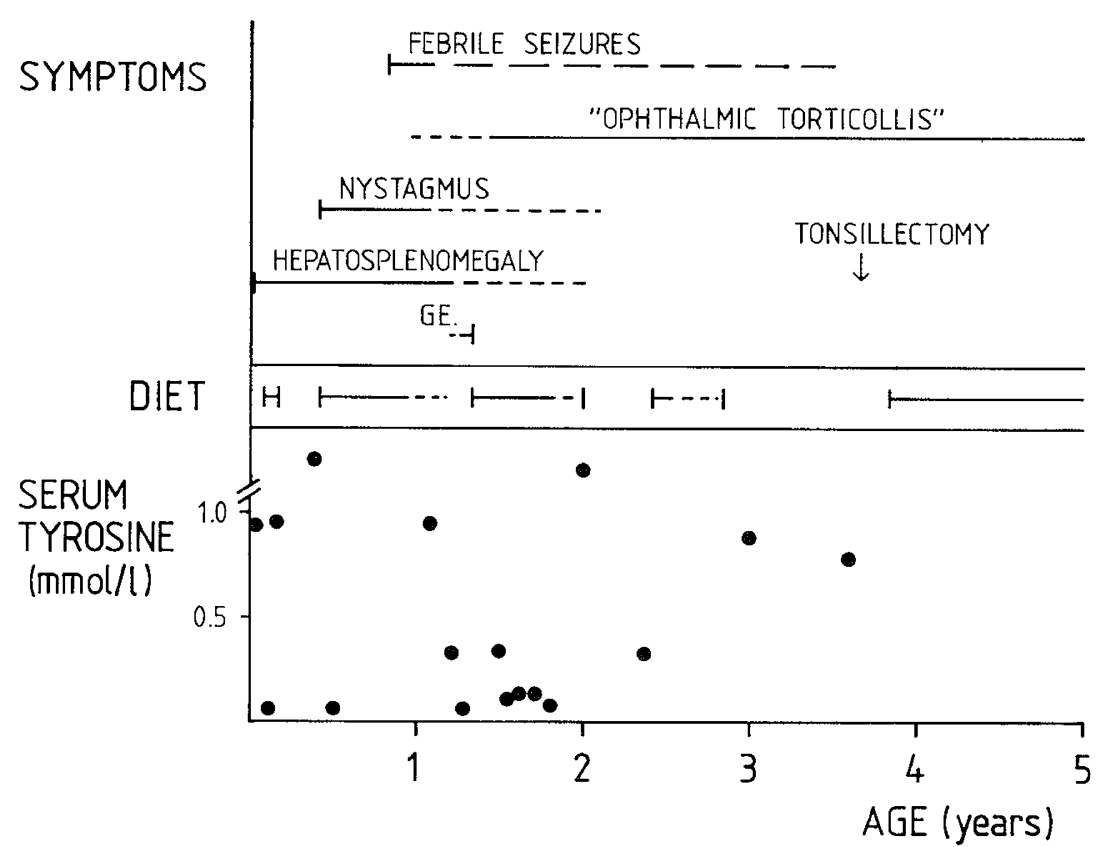

Fig. 1. Summary of clinical findings of the first patient in relation to diet and plasma tyrosine concentrations. $G E$, gastroenteritis.

seizures but the electroencephalography was normal on repeated occasions. A moderate psychomotor retardation observed during the first year of life was improved during the next few years. At the age of 5 , the boy has reached the maturity of a 4-year-old, showing a balanced profile.

During the first months of life, the boy had slightly elevated levels of serum transaminases. Liver biopsy according to Menghini was performed on four occasions: at the ages of 1 and 4 months, $1 \frac{1 / 2}{2}$ years, and 5 years. No histological changes could be revealed by light microscopic examination. The patient has not had any signs of hyperkeratosis or any other skin lesions.

The second patient, the younger sister of the first patient, was born without complications with a birth weight of $4200 \mathrm{~g}$. Neonatally, her plasma amino acid concentrations were normal and no organic acids were found in her urine. She was fed on normal formula and no check-ups were performed. At the age of 6 months, she was hospitalized because of infectious diarrhea. Three weeks after her recovery, she was found to be generally retarded in her psychomotor development. The liver was palpated 3-4 $\mathrm{cm}$ below the costal margin. She had elevated serum transaminases. Her plasma tyrosine concentration was slightly above normal level. No organic acids were found in the urine.

The actual investigation was performed at the age of 13 months. In her clinical status, a hepatomegaly was noticed and her psychomotoric development was generally somewhat retarded. Biochemically, she had normal serum transaminase levels and a markedly elevated serum tyrosine concentration. Needle biopsy of the liver showed no pathological changes under the light microscope. The electroencephalography was normal.

\section{BIOCHEMICAL STUDIES}

The boy had a plasma tyrosine concentration of $794 \mu \mathrm{mol} /$ liter (normal range, $39-77 \mu \mathrm{mol} /$ liter) at the age of $37 / 12$ years when he was not on the tyrosine-free diet. The plasma concentrations of the other amino acids were normal. The urinary excretion of tyrosine was $155 \mu \mathrm{mol} / 24 \mathrm{~h}$ (normal $<132 \mu \mathrm{mol}$ / $24 \mathrm{~h}$ ) and that of $p$-hydroxyphenylphenolic acids was $1.9 \mathrm{mmol} /$ $24 \mathrm{~h}$ (normal $<1.0 \mathrm{mmol} / 24 \mathrm{~h}$ ). Succinylacetoacetate and succinylacetone could not be demonstrated in the urine. Erythrocyte porphobilinogen synthetase and urinary porphobilinogen excretion were normal. These determinations were done as described in (22).

A liver biopsy by the method of Menghini was performed on the boy at the age of 5 years with the informed consent of the parents. The sample $(21.5 \mathrm{mg})$ was homogenized in 20 volumes of $320 \mathrm{mM}$ glycerol, $5 \mathrm{mM}$ potassium phosphate, $\mathrm{pH} 7.6$, using a Potter-Elvehjem glass-Teflon homogenizer. The homogenate was centrifuged at $20,000 \times g$ for $10 \mathrm{~min}$ and the supernatant fraction was kept frozen until analyzed. Tyrosine aminotransferase activity was assayed by a modification (31) of the method of Diamondstone (12) and aspartate aminotransferase activity and protein as described previously $(23,34)$. Total "tyrosine aminotransferase activity" of the supernatant fraction was 1.65 $\mathrm{nmol}$ of product $/ \mathrm{mg}$ of protein/min. For comparison, in a previous study from our laboratory, the corresponding activities in human fetuses of 12-22 weeks of gestational age averaged 0.53 and those for adults $19.8 \mathrm{nmol}$ of product $/ \mathrm{mg}$ of protein/ $\min (6)$. The activity is age-dependent in infancy but is on an adult level at the age of 5 (32). The electrofocusing pattern (31) of tyrosine aminotransferase activities from normal liver and from our patient are shown in Figures 2 and 3. In both cases, a peak of aspartate aminotransferase activity is seen at $\mathrm{pH}$ 9. These peaks also had low "tyrosine aminotransferase" activity as can be expected in the light of the broad substrate spectrum of this enzyme $(31,35)$. The enzyme is of no significance in normal metabolism of tyrosine because of its low affinity towards this nonphysiological substrate $(28,31,35)$. As shown in Figure 2, specific cytosolic tyrosine aminotransferase activity was divided into subforms at $\mathrm{pH} 5$ as expected $(4,31)$. A comparatively low peak (fractions 4-7) corresponding to cytosolic tyrosine aminotransferase is evident in Figure 3. The $K_{m}$ of these combined fractions towards tyrosine was $1.1 \mathrm{mM}$ (Fig. 4), which is close to the value obtained with purified enzyme (4). The corresponding determinations for fractions 5-9 (tyrosine aminotransferase) and 24-26 (mitochondrial aspartate aminotransferase) from the experiment shown in Figure 2 are displayed for comparison in Figure 4.

A liver biopsy was also obtained from the patient's younger sister at the age of 1 year. Due to the small size of the sample, the presence of specific tyrosine aminotransferase could not be demonstrated. Total tyrosine aminotransferase activity was clearly below that of the brother but the exact level could not be accurately determined.

\section{DISCUSSION}

The boy excreted neither succinylacetone nor succinylacetoacetate and his erythrocyte porphobilinogen synthetase activity was normal in clear distinction from tyrosinemia I. The clinical 


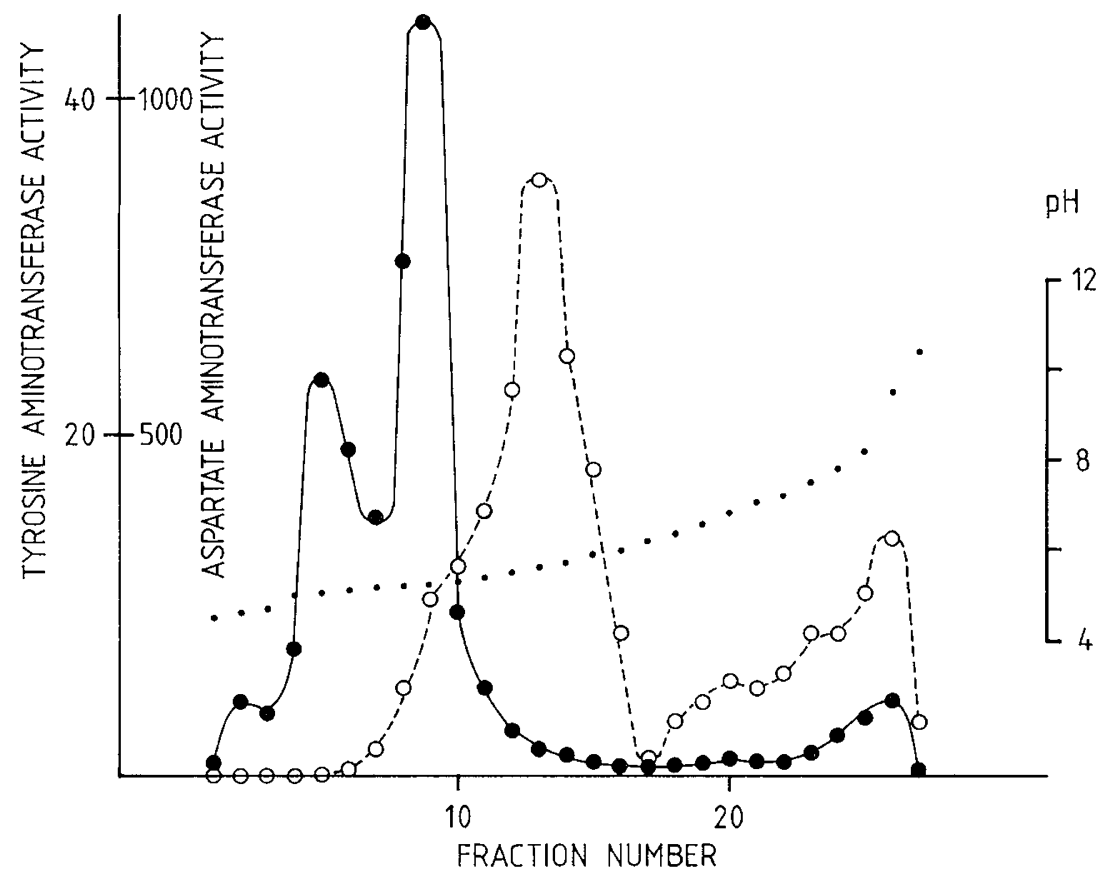

Fig. 2. Isoelectric focusing of normal human liver tyrosine aminotransferase activity. A sample of the liver of a healthy 32-year-old man (accidental death) was homogenized in 2 volumes of $320 \mathrm{mM}$ glycerol, and $5 \mathrm{ml}$ of the $100,000 \times g$ supernatant fraction was focused in a glycerol density gradient (total volume, $100 \mathrm{ml})$ in the $\mathrm{pH}$ range $4-6(1 \%$ Ampholine) for $40 \mathrm{~h}$ at $400 \mathrm{~V}$. Fractions were collected and analyzed for tyrosine aminotransferase and aspartate aminotransferase activities as described in the text. Enzyme activities are expressed as nanomoles of product/ml/ $\min .-$ tyrosine aminotransferase; $\mathrm{O}_{---} \mathrm{O}$, aspartate aminotransferase; $\cdots, \mathrm{pH}$.

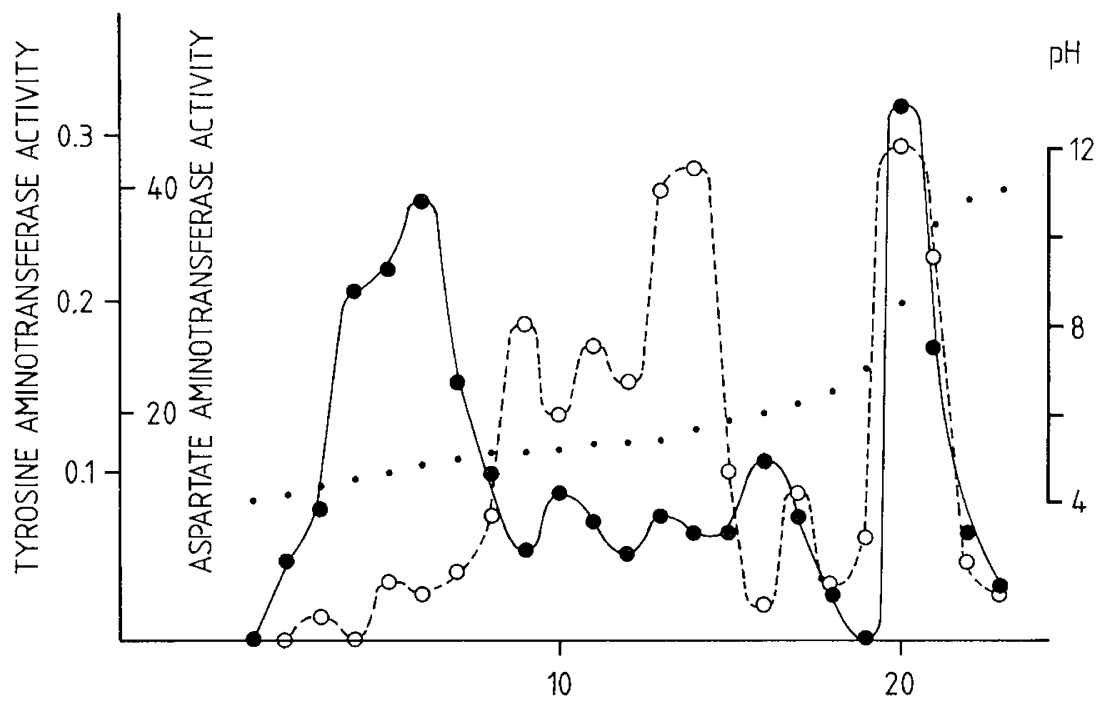

FRACTION NUMBER

Fig. 3. Isoelectric focusing of tyrosine aminotransferase activity from the liver of the first patient. Two hundred twenty-five $\mu$ l of the supernatant fraction described in the text were electrofocused in the $\mathrm{pH}$ range $4-6$ (1\% Ampholine) in a total volume of $15 \mathrm{ml}$ for $15 \mathrm{~h}$ at $400 \mathrm{~V}$. The symbols and units are as in Figure 2.

picture is different from tyrosinemia I as well as from RichnerHanhart syndrome in which palmar and plantar hyperkeratosis as well as corneal ulcerations and mental retardation are present. A case resembling those of our patients' has been described by Goldsmith et al. (16) who found lowered activity of tyrosine aminotransferase in the liver of an adult patient with tyrosinemia. Also, while this manuscript was in preparation, Giardini et al. (15) described a patient with tyrosinemia. Specific tyrosine aminotransferase was reported to be present in the patient's liver but the activity was low compared to normal ( $\mathrm{V}_{\max } 0.37$ versus 0.88 $\mu \mathrm{mol} / \mathrm{mg}$ of protein $/ \mathrm{h}$ ). $p$-Hydroxyphenylpyruvate dioxygenase activity was reported to be absent. Unfortunately, our samples were too small to allow determinations of this enzyme activity in our patients.

We have shown previously (6) that the specific enzyme tyrosine aminotransferase is present in fetal human liver at the end of the first trimester. However, the activity is low in fetal and premature neonatal human liver $(6,30)$. Although the enzyme is inducible by, e.g., glucocorticoids in organ culture of fetal liver, it cannot be induced by intraperitoneal injections of these hormones in utero in the rat. Therefore, it has been suggested that there is a factor in fetal liver that suppresses the induction by glucocorti- 

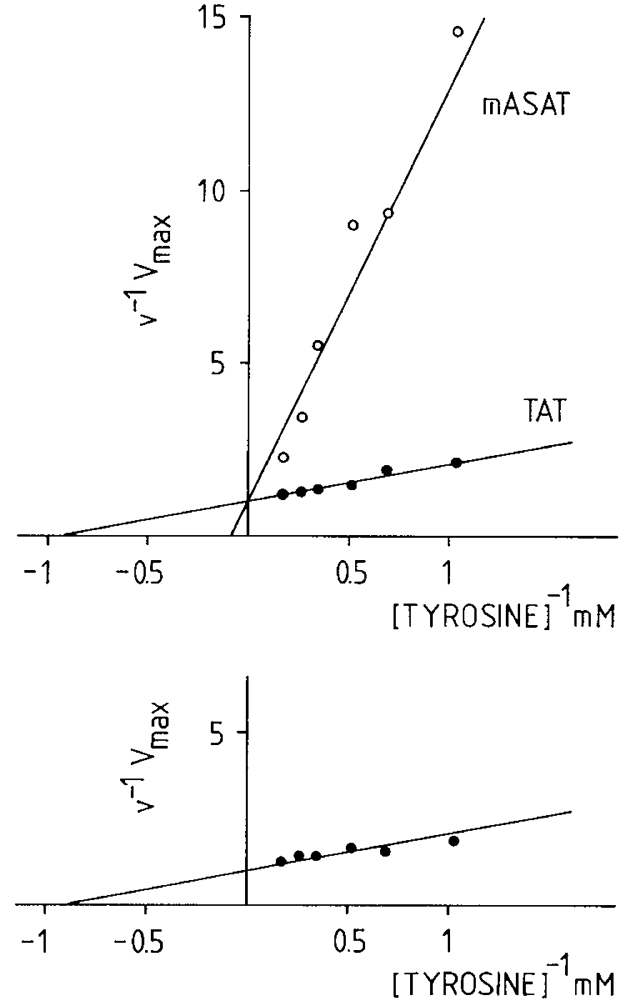

Fig. 4. Determination of $\mathrm{K}_{\mathrm{m}}$ values for tyrosine of specific tyrosine aminotransferase (TAT) from normal human liver (above) and from the liver of the first patient (below). See text for explanations. $m A S A T$, mitochondrial aspartate aminotransferase.

coids (37). In the case of our patients, the situation is similar to that in fetal and premature neonatal liver. They both have low liver tyrosine aminotransferase activities. It was shown, however, that the boy has a specific tyrosine aminotransferase that does not accept aspartate or oxaloacetate as substrate and has the same isoelectric point and $\mathrm{K}_{\mathrm{m}}$ value for tyrosine as the adult enzyme (4). Therefore, it is possible that the defect is not in the coding for tyrosine aminotransferase itself but in the regulatory mechanism of this enzyme.

\section{REFERENCES AND NOTES}

1. Andersson SM 1982 Induction of cytosolic tyrosine aminotransferase by dexamethasone in organ culture of fetal human liver. Early Hum Dev 6:165

2. Andersson SM 1983 Tyrosine aminotransferase. Ontogenic development and significance in tyrosinemia. Academic dissertation, University of Helsinki

3. Andersson SM 1983 Beta-adrenergic induction of tyrosine aminotransferase in organ culture of fetal rat and fetal human liver. Endocrinology 112:466

4. Andersson SM, Pispa JP 1982 Purification and properties of human liver tyrosine aminotransferase. Clin Chim Acta 125:117

5. Andersson SM, Räihä NCR, Ohisalo JJ 1980 Regulation of tyrosine aminotransferase in foetal rat liver. Biochem J 186:609

6. Andersson SM, Räihä NCR, Ohisalo JJ 1980 Tyrosine aminotransferase activ ity in human fetal liver. J Dev Physiol 2:17

7. Andersson SM, Salaspuro M, Ohisalo JJ 1982 Metabolic basis of hypertyrosinemia in liver disease. Gastroenterology 82:554

8. Berger R, Smit GPA, Stoker-de Vries SA, Duran M, Ketting D, Wadman SK 1981 Deficiency of fumarylacetoacetase in a patient with hereditary tyrosinemia. Clin Chim Acta 114:37

9. Berry HK 1976 Tyrosinemia. In: Ampola M (ed): Clinics in Perinatology. Early Detection and Management of Inborn Errors. Philadelphia, WB Saunders, pp 31-40

10. Christensen E, Brock Jacobsen B, Gregersen N, Hjeds H, Pedersen JB, Brand NJ, Baekmark UB 1981 Urinary excretion of succinylacetone and $\delta$-amino- levulinic acid in patients with hereditary tyrosinemia. Clin Chim Acta $116: 331$

11. Coufalik AH, Monder C 1980 Regulation of tyrosine oxidizing system in fetal rat liver. Arch Biochem Biophys 199:67

12. Diamondstone TI 1966 Assay of tyrosine aminotransferase activity by conversion of $p$-hydroxyphenylpyruvate to $p$-hydroxyphenylbenzaldehyde. Anal Biochem 16:395

13. Dickson AJ, Marston FAO, Pogson Cl 1981 Tyrosine aminotransferase as the rate limiting step for tyrosine catabolism in isolated rat liver cells. FEBS Lett 127:28

14. Fellman JH, Vanbellinghen PJ, Jones RI, Koler RD 1969 Soluble and mitochondrial forms of tyrosine aminotransferase. Relationship to human tyrosinemia. Biochemistry 8:615

15. Giardini O, Cantani A, Kennaway NG, d'Eufemia P 1983 Chronic tyrosinemia associated with 4-hydroxyphenylpyruvate dioxygenase deficiency with acute intermittent ataxia and without visceral and bone involvement. Pediatr Res $17: 25$

16. Goldsmith LA 1978 Molecular biology and molecular pathology of a newly described molecular disease-tyrosinemia II (the Richner-Hanhart syndrome). Exp Cell Biol 46:96

17. Goldsmith LA 1983 Tyrosinemia and related disorders. In: Stanbury JB, Wyngaarden JB, Fredrickson DS, Goldstein JL, Brown MS (eds): The Metabolic Basis of Inherited Disease, 5th ed. New York, McGraw-Hill, pp 287-299

18. Goldsmith LA, Thorpe J, Roe CR 1979 Hepatic enzymes of tyrosine metabolism in tyrosinemia II. J Invest Dermatol 73:530

19. Heaton JH, Shilling EE, Gelehrter TD, Rechler MM, Spencer CJ, Nissley SP 1980 Induction of tyrosine aminotransferase and amino acid transport in rat hepatoma cells by insulin and the insulin-like growth factor, multiplication-stimulating activity. Biochim Biophys Acta 632:192

20. Kennaway NG, Buist NRM, Fellman JH 1972 The origin of urinary $p$ hydroxyphenylpyruvate in a patient with hepatic cytosol tyrosine aminotransferase deficiency. Clin Chim Acta 41:157

21. Lemonnier F, Charpentier C, Odievre M, Larregue M, Lemonnier A 1979 Tyrosine aminotransferase isoenzyme deficiency. J Pediatr 94:931

22. Lindblad B, Lindstedt S, Steen G 1977 On the enzymatic defects in hereditary tyrosinemia. Proc Natl Acad Sci USA 74:4641

23. Lowry OH, Rosebrough NJ, Farr AL, Randall RJ 1951 Protein measurement with the Folin phenol reagent. J Biol Chem 193.265

24. Mamunes P, Prince PE, Thornton NH, Hunt PA, Hitchcock ES 1976 Intellectual deficits after transient tyrosinemia in the term neonate. Pediatrics 57:675

25. Marston FAO, Dickson AJ, Pogson CI 1981 Factors affecting induction of tyrosine aminotransferase in isolated rat liver cells. Mol Cell Biochem 34:59

26. Morgan MY, Milsom JP, Sherlock S 1978 Plasma ratio of valine, leucine and isoleucine to phenylalanine and tyrosine in liver disease. Gut 19:1068

27. Ohisalo JJ 1977 Liver tyrosine aminotransferase. Academic dissertation, University of Helsinki

28. Ohisalo JJ, Andersson BM, Viljanen AA, Andersson SM 1982 Is there a brain tyrosine aminotransferase? Biochem $J$ 204:62

29. Ohisalo $J J$, Hassinen IE, Pispa JP 1974 On the induction of tyrosine aminotransferase in rat liver by $\alpha$-methyl-p-tyrosine. Biochim Biophys Acta 362:48

30. Ohisalo JJ, Laskowska-Klita T, Andersson SM 1982 Development of tyrosine aminotransferase and $p$-hydroxyphenylpyruvate dioxygenase activities in fetal and neonatal human liver. J Clin Invest 70:198

31. Ohisalo JJ, Pispa JP 1976 Heterogeneity of hepatic tyrosine aminotransferase. Separation of the multiple forms from rat and frog liver by isoelectric focusing and hydroxylapatite column chromatography and their partial characterization. Acta Chem Scand B 30:491

32. Räihä NCR, Schwartz AL, Lindroos MC 1971 Induction of tyrosine- $\alpha$-ketoglutarate transaminase in fetal rat and fetal human liver in organ culture. Pediatr Res 5:70

33. Rehak A, Selim MM, Yadav G 1981 Richner-Hanhart syndrome (tyrosinemia2) (report of four cases without ocular involvement). Br J Dermatol 104:469

34. Reitman A, Frankel S 1957 A colorimetric method for the determination of serum glutamic oxaloacetic and glutamic pyruvic transaminases. Am J Clin Pathol 28:56

35. Rej R 1976 Isolation and characterization of the aspartate aminotransferase isoenzyme system from human liver. Academic dissertation, Albany Medical College,

36. Weinberg AG, Mize CE, Worthen HG 1979 The occurrence of hepatoma in the chronic form of hereditary tyrosinemia. J Pediatr 88:434

37. Yeoh GCT, Arbuckle T, Oliver IT 1979 Tyrosine aminotransferase induction in hepatocytes cultured from rat foetuses treated with dexamethasone in utero. Biochem J 180:545

38. This research was supported by grants from the Swedish Research Counci (5361) and by The Magnus Ehrnrooth Foundation.

39. Requests for reprints should be addressed to: Sture Andersson, M.D., Department of Medical Chemistry, Siltavuorenpenger 10, 00170 Helsinki 17 , Finland.

40. Received for publication May 2, 1983

41. Accepted for publication October 13,1983 